\title{
Gamma-Band Activation Predicts Both Associative Memory and Cortical Plasticity
}

\author{
Drew B. Headley and Norman M. Weinberger \\ Center for the Neurobiology of Learning and Memory, Department of Neurobiology and Behavior, University of California, Irvine, California 92697-3800
}

Gamma-band oscillations are a ubiquitous phenomenon in the nervous system and have been implicated in multiple aspects of cognition. In particular, the strength of gamma oscillations at the time a stimulus is encoded predicts its subsequent retrieval, suggesting that gamma may reflect enhanced mnemonic processing. Likewise, activity in the gamma-band can modulate plasticity in vitro. However, it is unclear whether experience-dependent plasticity in vivo is also related to gamma-band activation. The aim of the present study was to determine whether gamma activation in primary auditory cortex modulates both the associative memory for an auditory stimulus during classical conditioning and its accompanying specific receptive field plasticity. Rats received multiple daily sessions of single tone/shock trace and two-tone discrimination conditioning, during which local field potentials and multiunit discharges were recorded from chronically implanted electrodes. We found that the strength of tone-induced gamma predicted the acquisition of associative memory $24 \mathrm{~h}$ later and ceased to predict subsequent performance once asymptote was reached. Gamma activation also predicted receptive field plasticity that specifically enhanced representation of the signal tone. This concordance provides a long-sought link between gamma oscillations, cortical plasticity, and the formation of new memories.

\section{Introduction}

Coordinated activity is ubiquitous in the nervous system. Populations of neurons coactivate repeatedly for short temporal epochs, resulting in synchronized oscillations expressed by unit activity and local field potentials (LFP). These oscillations have been linked to behavioral performance and physiological functioning (Buzsáki, 2006). Presently, oscillations in the gamma band $(40-120 \mathrm{~Hz})$ are receiving much attention for their possible role in memory and neural plasticity.

Induced gamma ( $>100 \mathrm{~ms}$ latency following stimulus onset) is relevant to behavioral memory because its strength is enhanced during short-term memory tasks (Lutzenberger et al., 2002; Pesaran et al., 2002). Notably, this enhancement predicts subsequent recall; stimuli that are later recalled display enhanced gamma power during encoding (Fell et al., 2001; Osipova et al., 2006; Sederberg et al., 2006, 2007). Furthermore, strength of recall is positively correlated with coherence between spiking and the LFP in the gamma band (Jutras et al., 2009).

Gamma oscillations are also prime candidates for modulating synaptic plasticity because they reflect coordinated activity on the time scale of excitatory postsynaptic responses and are within the range of relative spike timing that is optimal for spike timing-

Received May 19, 2011; revised June 14, 2011; accepted July 19, 2011

Author contributions: D.B.H. and N.M.W. designed research; D.B.H. performed research; D.B.H. analyzed data; D.B.H. and N.M.W. wrote the paper.

This research was supported by research Grant DC-02938 from the National Institutes of Health/National Institute on Deafness and 0ther Communication Disorders to N.M.W. We thank Jacquie Weinberger and Gabriel Hui for secretarial assistance.

The authors declare no competing financial interests.

Correspondence should be addressed to Dr. Norman M. Weinberger, Center for the Neurobiology of Learning and Memory, University of California, Irvine, CA 92697-3800. E-mail: nmweinbe@uci.edu.

DOI:10.1523/JNEUROSCI.2528-11.2011

Copyright $\odot 2011$ the authors $\quad 0270-6474 / 11 / 3112748-11 \$ 15.00 / 0$ dependent plasticity (10-20 ms) (Bi and Poo, 1998). The dependence of synaptic plasticity on gamma-band activity has been tested in vitro for the hippocampus. The direction of synaptic plasticity, either potentiating (LTP) or depressing, is determined by the timing of afferent stimulation relative to the phase of ongoing gamma oscillations (Wespatat et al., 2004). Furthermore, LTP is preferentially induced by naturalistic patterns of spiking that display gamma periodicity (Isaac et al., 2009).

Although these intriguing findings suggest that gamma-band activation is involved in plasticity that underlies memory formation, it is not yet known whether gamma affects in vivo experiencedependent plasticity, particularly plasticity that has been linked to memory.

Understanding gamma's relationship to behavioral memory and neural plasticity would be advanced by their simultaneous study in the same subjects under the same training procedure. Auditory fear conditioning is a well established learning situation in which a tone that predicts shock induces behavioral memory accompanied by highly specific plasticity in the primary auditory cortex (A1). Typified by receptive field (RF) shifts toward the conditioned stimulus (CS) frequency, it is rapidly formed, consolidates, and is maintained for at least 2 months (Weinberger, 2004). Local synaptic plasticity appears to support RF shifts, with both extracellular evoked potentials and intracellularly recorded synaptic potentials displaying enhanced strength after a tone was paired with a shock or nucleus basalis stimulation, respectively (Galván and Weinberger, 2002; Froemke et al., 2007). Moreover, the CS frequency representational area within the tonotopic map is increased and the amount of enhanced representation predicts both its level of acquired behavioral importance (Rutkowski and Weinberger, 2005) and its memory strength (Bieszczad and Weinberger, 2010). Together, these findings indicate that 
RF plasticity in A1 is a likely substrate for auditory associative memory.

We investigated the relationship between gamma-band activity and both memory and cortical RF plasticity during fear conditioning. Gamma-band activity elicited by the training stimuli was tracked across multiple days of single-tone and two-tone discrimination training while obtaining frequency RFs in A1 and behavioral evidence of memory.

\section{Materials and Methods}

Subjects. The subjects were 23 adult male Sprague-Dawley rats (275-350 g) (Charles River). The data from two were excluded after histological examination revealed damage of the auditory cortex caused by the implanted electrode array. Animals were housed individually with ad libitum food and water, on a 12/12 h light-dark cycle (lights on at 7:00 A.M.). All surgical and experimental treatments were approved by the Institutional Animal Care and Use Committee for the University of California at Irvine.

Surgical procedures. Subjects were anesthetized with sodium pentobarbital $(55 \mathrm{mg} / \mathrm{kg}$, i.p.; Sigma-Aldrich) and atropine (0.1 mg, i.m.; MedPharmex) and then placed in a stereotactic frame (Kopf) using blunt ear bars. A thermostatically controlled heating pad maintained body temperature. Lidocaine (AstraZeneca) was injected into the scalp subcutaneously, followed by a midline incision. The scalp was retracted and the periosteum was removed. Miniature stainless steel screws $(\# 0-80$, Small Parts) were inserted into the calvaria. One of the screws served as a ground. Dental acrylic was applied to the screws, forming the base of a pedestal. The right temporal muscle was resected and screws were inserted into the temporal and parietal bones to anchor an electrode array that was mechanically independent from the main pedestal. The electrode array consisted of polyimide-coated $50 \mu \mathrm{m}$ tungsten wires (California Fine Wire) arranged in a grid pattern of either one row of four or two rows of eight, evenly spaced either 200 or $400 \mu \mathrm{m}$. A craniotomy $(\sim 3 \times 2 \mathrm{~mm})$ was made overlying the primary auditory field using skull landmarks. The electrode array was centered on A1 using vascular landmarks and noise burst evoked potentials recorded from the cortical surface. Electrodes were inserted manually through the dura to a depth of $\sim 800 \mu \mathrm{m}$, at which point multiunit unit activity was reliably present. To further confirm that placement was in $\mathrm{Al}$, we verified that these units were driven by tonal stimuli at short latency $(<20 \mathrm{~ms})$, and that the best frequency across electrodes increased in the caudal to rostral direction. Then the craniotomy was covered with gelfoam and dental acrylic, which anchored the array to the skull.

The electrocardiogram was obtained from an electrode sutured subcutaneously around the thoracic musculature, either after implantation of the array or $5 \mathrm{~d}$ later. EKG recordings were obtained from a Tefloncoated stranded stainless steel wire (\#793200, A-M Systems) with a patch of insulation removed where the wire would directly oppose the heart. A separate subcutaneous loop of wire was placed over the back to serve as the ground. Both wires were previously soldered into a pin connector, which was then embedded in the skull pedestal. To provide anchorage for limiting head movement during training, metal loops ( $\sim 5 \mathrm{~mm}$ radius) were added to the pedestal.

Experimental enclosure. The experimental enclosure consisted of a wire mesh alley $(30.5 \times 5.8 \times 6.0 \mathrm{~cm})$ that tilted $\sim 20^{\circ}$ at the base, such that the subject's head was higher than its body. The enclosure was kept within an acoustic isolation chamber (Industrial Acoustics). Rats entered the cage from the rear, crawled up to the closed end at the front, and had their head movement restricted to $\sim 15^{\circ}$ in any direction by elastic bands connecting the metal loops on their pedestals to fixation points on the frame of the enclosure. Subjects did not hesitate to enter the enclosure or to having their heads restrained. This setup practically eliminated all head movement, except for occasional grooming and voluntary postural adjustments. It also maintained wakefulness and a consistent relationship between an externally placed speaker and the head.

Before surgery, subjects typically received two $\sim 1 \mathrm{~h}$ acclimation sessions to the training cage/acoustic chamber and to tones used to probe cortical frequency tuning. Each session was divided into two periods: first
( $\sim 30 \mathrm{~min}$ ), tones were presented with the training chamber lights on; second, there was a silent period ( $\sim 30 \mathrm{~min})$ with the lights off. After $5-7$ $\mathrm{d}$ of recovery from surgery, subjects underwent at least one more acclimation session, but with head restraint added. Rats rapidly ( $<10 \mathrm{~min})$ adjusted to the head restraint.

Acoustic calibration and stimuli. An electromagnetic speaker (FF1, Tucker-Davis Technologies) was fixed parallel to the axis of the ear canal, $\sim 15 \mathrm{~cm}$ from the left ear. Its frequency transfer function was determined using a tone generator (RP2.1, Tucker-Davis Technologies), calibrated microphone, and preamplifier (Brüel and Kjær). A programmable attenuator (PA5, Tucker-Davis Technologies) was used to equalize the speaker output across all tone frequencies.

Frequency receptive fields were obtained using pure tones, consisting of $50 \mathrm{~ms}$ pure tone pips $(0.75-48.0 \mathrm{kHz}, 50 \mathrm{~dB}$ SPL, $8 \mathrm{~ms}$ rise/fall time), presented through a calibrated speaker system (RP2.1 and PA5, TuckerDavis Technologies). Training stimuli were identical pure tones, except with a duration of $10 \mathrm{~s}$.

Receptive field acquisition. At the start of each training session, $10 \mathrm{~min}$ after subjects were placed in the cage, a battery of stimuli (pure tones and spectrotemporal ripples) was presented to assess tuning. For this paper, only receptive fields obtained using pure tones are presented. All subjects received a similar cohort of stimuli, and for each subject the same stimuli were presented every day.

Twenty repetitions of each tone frequency were delivered, with tones of different frequencies randomly intermixed. To keep the RF acquisition and training contexts distinct, RFs were obtained with the chamber lights on, whereas training occurred in the dark. This minimized the possibility that RF plasticity reflected attentional modulation, which can be produced by the training context (Diamond and Weinberger, 1989; Fritz et al., 2003).

Training. After the RF acquisition period, the chamber lights were turned off and training was performed in the dark. Two training phases were used in this study (Fig. $1 \mathrm{~A}$ ). The first was a tone/shock classical conditioning phase, with subjects receiving $2-8$ (median $=4$ ) daily sessions. After the classical conditioning phase, subjects underwent 1-6 (median $=4$ ) sessions of two-tone discrimination conditioning (two subjects did not receive discrimination training). Discrimination training established that conditioned responding (CR) was associative and not driven by either sensitization (repeated exposure to a CS) or pseudoconditioning (repeated exposure to an unconditioned stimulus) (Bouton, 2006). All training sessions were separated by at least $1 \mathrm{~d}$, and almost all $(\sim 98 \%)$ were performed on sequential days. For each subject, care was taken to keep the session starting times consistent across days. The first four sessions of conditioning and discrimination were analyzed, as they yielded the most data.

All subjects had similar training session durations lasting $1.5 \mathrm{~h}$ on average, but with different numbers of trials. Eleven subjects received 10 trials per day [ $8 \mathrm{~min}$ mean intertrial interval (ITI)], and 10 had 40 trials (2 min mean ITI). The median number of sessions was not significantly different between groups, either for conditioning (Group $10=4.0$, Group $40=4.0$; Wilcoxon rank-sum, $W=126.5, p>0.1$ ) or discrimination (Group $10=4.0$, Group $40=4.0 ; W=105, p>0.5$ ). Moreover, the groups were also similar in the frequencies chosen for the CS+ (reinforced tone) and CS - (unreinforced tone), cardiac responses to the $\mathrm{CS}+$ or CS - , and overall mean gamma strength. Therefore, the two groups were combined for all other analyses.

Figure $1 C$ illustrates the arrangement of stimuli during training. For classical conditioning, a trial consisted of a $10 \mathrm{~s}$ tone (CS+), followed by a $1.0 \mathrm{~s}$ silent trace, and then a $1.0 \mathrm{~s}$ shock. A different CS+ and CSfrequency was chosen for each subject, with some RFs tuned near it, whereas others were tuned farther away. The same tone frequency was used on all training days. Shock $(40 \mathrm{~Hz}$, biphasic 8.3 ms pulses, constant current source; \#H13-15, Coulbourn Instruments) was delivered through the EKG electrodes. It was adjusted during the first conditioning session to the minimal possible level that elicited a brief reflexive body contraction; shocks never elicited struggling or escape responses. Good conditioning could be achieved with weak shock because of the high degree of sensitivity of the cardiovascular system during learning (Winters et al., 2002). 


\section{A Experimental phases}

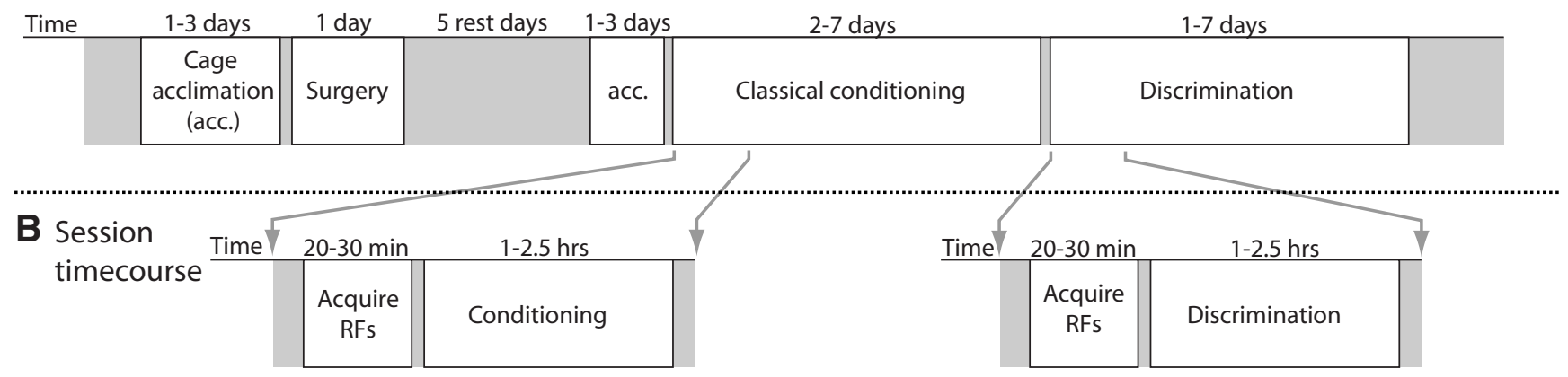

C Trial
structure

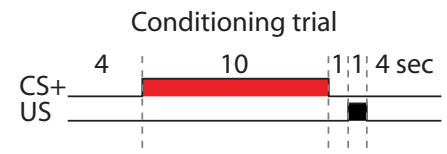

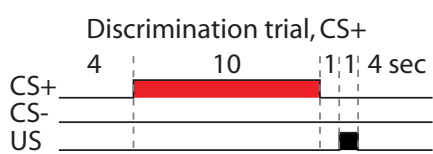

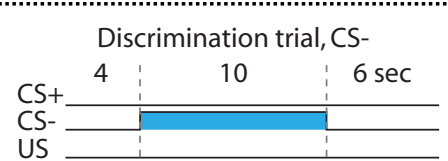

Figure 1. Overview of the experimental design. $A$, Subjects received several different phases of training. First they were acclimated to the training context for several days before and after surgery. This reduced their stress during subsequent training. After the acclimation phase, aversive classical conditioning with a tone and shock took place over several days, which is known to induce both conditioned responding to the tone and plasticity in A1. The last phase was discrimination training, which allowed us to test whether subjects had formed an associative memory for the CS.$+ B$, Each of the conditioning and discrimination sessions could be divided into two phases. RFs were acquired by playing a series of tone pips, followed by the training phase. $C$, Trials featuring the CS + were the same for both conditioning and discrimination; the $C S+$ was presented for $10 \mathrm{~s}$, followed by a $1 \mathrm{~s}$ silent trace period, and then a $1 \mathrm{~s}$ shock. During discrimination, a $10 \mathrm{~s} C S-$ was presented for half the trials, without any reinforcement. US, Unconditioned stimulus.

Discrimination training was begun usually after four sessions of classical conditioning, by which point subjects had well acquired the tone/ shock association. The unreinforced tone (CS-) was easily discriminable from the CS+, being at least 1 octave away from that frequency used in conditioning. CS + and CS - trials were given with equal probability, pseudorandomly intermixed, with at most three successive trials of the same type. Discrimination training typically lasted $4 \mathrm{~d}$.

Heart rate acquisition and analysis. EKG signals were amplified $(\times 10,000)$, filtered $(10-300 \mathrm{~Hz}$; model EX-1000, Dagan), digitized at $610 \mathrm{~Hz}$ and stored (RP2.1, Tucker-Davis Technologies). Heartbeats were threshold edge-detected "R-wave" events (peak voltage of individual heartbeats). Beat times were converted to beats per minute and linearly interpolated at the sampling rate of the original EKG signal.

The conditioned response (CR) on each trial was the percentage change in mean heart rate between a $4 \mathrm{~s}$ pre-CS period and the entire $10 \mathrm{~s}$ CS period (Fig. 2A). The cardiac conditioned response in fear conditioning is typically bradycardia (slowing of heart rate) when animals are confined (Teyler, 1971; Fendt and Fanselow, 1999). CS-evoked bradycardia is sensitive to floor effects; its magnitude is reduced when the heart rate is already low before the tone. To eliminate this problem, analysis of heart rate was restricted to trials for which baseline heart rate was in the upper 50th percentile.

Neural data acquisition. Both unit activity and LFPs were recorded after amplification (unity gain, LP16CH headstage, RA16 digitizer with 4 or 8 channel capacity, Tucker-Davis Technologies). LFPs were obtained offline with a $0.01-300 \mathrm{~Hz}$ second-order bandpass Butterworth filter using the MATLAB "filtfilt" command. LFPs were referenced to the ground screw in the calvaria. Movement artifacts were identified by visual inspection, and all trials containing such artifacts were eliminated from further analysis $(<4.5 \%)$. Subsequent spectral analyses were performed only on recording sites that met several standard criteria: (1) displayed frequency tuned evoked potentials (EP) to auditory stimuli, (2) had an initially negative EP waveform, and (3) with an onset latency $<30 \mathrm{~ms}$ (e.g., Goldstein et al., 1970; Reale and Imig, 1980; Sally and Kelly, 1988).

Unit activity (Butterworth filter, $300-3000 \mathrm{~Hz}$ second-order bandpass) was detected and sorted offline. Units were detected with a window discriminator that selected spikes with a negative peak exceeding -2.5 root mean square (RMS) followed within $0.6 \mathrm{~ms}$ by a positive peak $>2.0$ RMS. Movement and other artifacts were subtracted out by referencing to an electrode that rested on the cortical surface. All reported unit activity is multiunit.

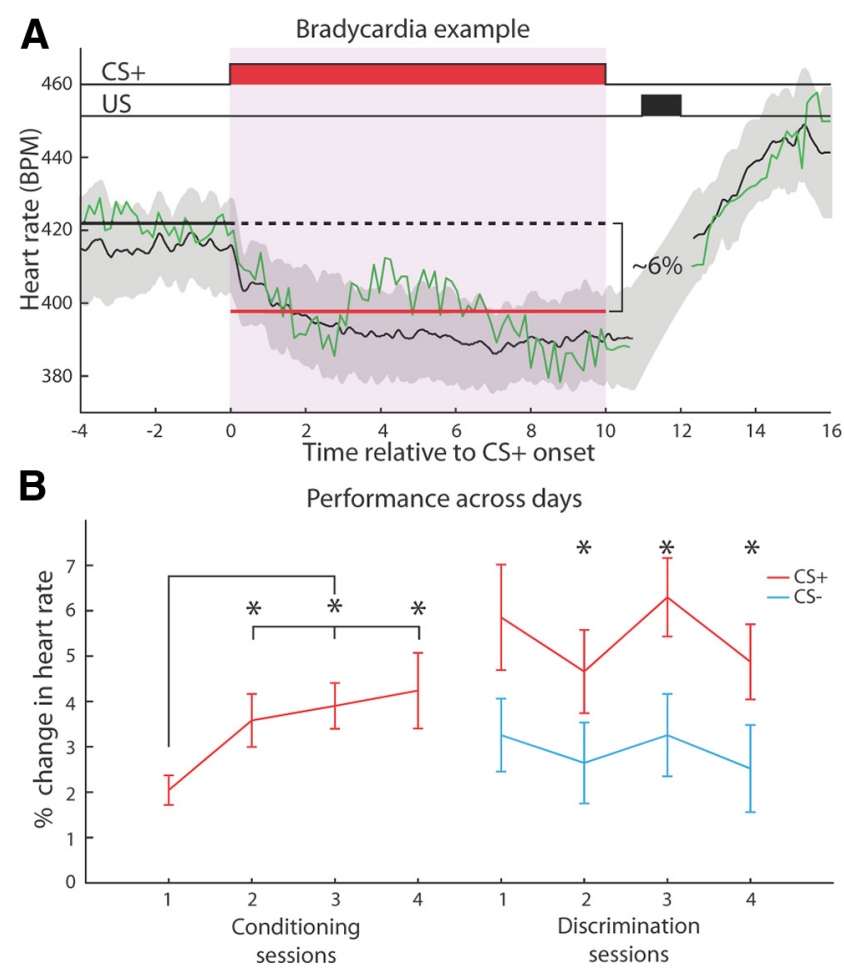

Figure 2. Behavior during training. $A$, An example of conditioned bradycardia from a single subject on the second day of conditioning. The green line is a single trial, and the black line and gray shading are the mean CR across all valid trials for that session and \pm 1 SD, respectively. The thick solid black line denotes the pre-CS baseline period, and the red line demonstrates the CS period used to calculate the $C R$ strength. For each trial, the $C R$ was defined as the percentage difference between the mean heart rate for each of these periods. US, Unconditioned stimulus. $\boldsymbol{B}$, Subjects learned the tone/shock contingency within a single day of training, displaying asymptotic performance by the second day of training. They exhibited evidence of discrimination between the $\mathrm{CS}+$ and $\mathrm{CS}$ - on the first day of training, and a within-session statistically significant difference between the responses to either stimulus was attained on the second day of training. Across all discrimination days, the $C S+$ elicited a significantly stronger $C R$ than the CS-. Asterisks denote a significant difference (Wilcoxon rank-sum test). Error bars indicate mean \pm SEM 
Analysis of frequency receptive fields. For each tone frequency, we calculated the average firing rate during an evoked response window, minus the firing rate for a $50 \mathrm{~ms}$ pretone window. The response window was separately determined for each electrode and kept constant across all days. The response window was calculated as the time when the mean firing rate to all tone pips across all recorded days exceeded 2.0 SDs of the $50 \mathrm{~ms}$ pretone spontaneous period. To ensure that all analyzed receptive fields were sufficiently above threshold, we required that each contained at least two adjacent frequencies whose response magnitudes were minimally twice the mean $\mathrm{SD}$ of responses to all frequencies composing that RF. If an electrode's RF failed to meet the above criteria for a given session, then that electrode was excluded from RF analysis for all subsequent sessions.

Several steps were taken to calculate RF changes. Each RF was normalized by dividing by the maximum response, so the best frequency (BF) had a response strength of 1.0. The RF change $(\Delta R F)$ for each training day was taken relative to the same pretraining baseline RF, obtained on the first training day. For the baseline RF, if the mean response to a particular frequency was less than half of the SD of responses at that frequency, it was removed from analysis as unreliable. To assess plasticity, an electrode had to have a valid RF at least for the first and second training sessions (baseline and posttraining, respectively).

Spectral analysis. LFP spectrograms for each training trial were calculated using the Chronux package for MATLAB (MathWorks). Its theoretical basis (Thomson, 1982) and application (Bokil et al., 2010) have been described previously, so our discussion will be brief. The Chronux package performs spectral analysis with a multitaper technique for windowing a time series. Traditional spectral analysis relies on a single windowing function, which can result in a high degree of variance for spectral estimates and an uncontrolled bias in the estimation of particular frequency bands. To help alleviate these issues, a collection of windowing functions can be used that provide multiple estimates of spectral power and with each tailored for estimating spectral power with a constant passband bandwidth. Chronux uses a collection of discrete prolate spheroidal functions [for this study, the time-bandwidth product $(\mathrm{TW})=3$, and the number of tapers $(\mathrm{K})=5$ ] to calculate multiple estimates of spectral power for a particular time window and frequency band. The LFP trace was sampled with a succession of $200 \mathrm{~ms}$ windows, each overlapping by $100 \mathrm{~ms}$.

Before calculating the strength of gamma elicited by the CSs, spectrograms were normalized. Each frequency band of the spectrogram was $z$-normalized to the mean and SD of the $4.0 \mathrm{~s}$ baseline period preceding trial onset (Fig. 3A; for an example trial, see Fig. 3B). All further analyses were performed on the normalized spectrogram. The power in the gamma band (40-120 Hz, Fig. 3A; Chrobak and Buzsáki, 1998; M. Siegel and König, 2003) at a given time point was defined as the 90th percentile of spectral power across the entire gamma band. The central role of acetylcholine (ACh) in modulating both cortical gamma oscillations (Rodriguez et al., 2004) and Al receptive field plasticity (Weinberger, 2004) led us to analyze a particular timeframe of CS-induced gamma. For correlating induced gamma with conditioned responding or RF plasticity, the strength of induced gamma was quantified as the median gamma power 2-4.0 s after CS onset. This time window corresponds to the onset of ACh release (Parikh et al., 2007). However, our results were robust to shifting that time window from 1 to $5.0 \mathrm{~s}$ after CS onset.

Statistical analyses. Statistical significance was evaluated with rankbased nonparametric tests (Siegel and Castellan, 1988). ANOVAs were used to assess group effects in certain circumstances. Spearman's correlation coefficient (a rank-based nonparametric test of correlation) was used to determine whether a significant monotonic relationship was present between two variables. A Wilcoxon rank-sum test for equal medians determined whether two groups were significantly different, and a tail hypothesis test was used for certain circumstances that warranted a specific a priori prediction (e.g., CS $+\mathrm{CR}>\mathrm{CS}-\mathrm{CR}$ ). To evaluate whether a distribution's median was significantly different from zero (as would be the case for RF plasticity), we used a Wilcoxon signed-rank test for zero median. All statistical analyses were performed in either MATLAB or R (version 2.12.1, R Foundation for Statistical Computing).

\section{Results}

\section{Behavior}

Subjects developed fear conditioning, that is, an association between the tonal CS + and the shock during the first stage of training. Figure $2 \mathrm{~A}$ presents a typical example of conditioned bradycardia from Day 2 . The CS + elicited a clear and consistent reduction in heart rate during its $10 \mathrm{~s}$ presentation. Figure $2 \mathrm{~B}$ presents group data for $4 \mathrm{~d}$ of conditioning and discrimination training. CRs developed within the first conditioning session, reached statistical significance on the next day, and persisted for the remaining sessions. Asymptotic performance was reached on Day 2, as indicated by the fact that the magnitude of the CR on the first day was significantly less than that for the following three sessions (Wilcoxon rank-sum one-tailed, Day 2: $n=21, W=$ 136, $p<0.05$; Day 3: $n=20, W=111, p<0.01$; Day 4: $n=18$, $W=122, p<0.05)$, which did not differ among themselves (Wilcoxon rank-sum, 2 vs 3 : $W=196, p>0.5 ; 2$ vs 4 : $W=178$, $p>0.5 ; 3$ vs $4: W=185, p>0.5)$.

During discrimination training, bradycardia to the CS + was significantly larger than to the CS - across the $4 \mathrm{~d}$ of training (Fig. $2 B$; Wilcoxon rank-sum, $n=68, W=3073, p<0.001)$. This difference attained statistical significance on Day 2 of discrimination, and it was maintained on Days 3 and 4 (Fig. $2 B$; Wilcoxon rank-sum one-tailed, Day 1: $n=19, W=227, p>0.05$; Day 2: $n=17, W=193, p<0.05$; Day 3: $n=18, W=210, p<0.05$; Day 4: $n=14, W=143, p<0.05)$. Successful discrimination indicated that conditioned bradycardia was mediated by an associative process, rather than by sensitization or pseudoconditioning.

\section{Relationship between gamma and long-term memory}

Multiple recording sites in A1 displayed activation in the gamma band to the CS + . This activation was typified by a brief evoked response within $200 \mathrm{~ms}$ of tone onset, followed by a longer lasting induced response (Fig. $3 A$ ). For each subject, we calculated the mean strength of gamma induced by the CS + across all sites in A1. This was done for each daily training session, providing readout of a subject's induced gamma strength across days.

To investigate the relationship between gamma and longterm memory, we compared CS +-induced gamma during a session with performance on the subsequent session, $24 \mathrm{~h}$ later. This comparison was motivated by the literature on "subsequent memory effects" (Introduction), showing that neural activity evoked by a stimulus at the time of encoding can be correlated with subsequent memory for that same stimulus (Paller and Wagner, 2002). In the present study, CS+-induced gamma during training was the neural signature during encoding, and conditioned responding to the same CS+ on the following day indicated the strength of subsequent memory.

Gamma activation $\left(90^{\text {th }}\right.$ percentile of power in the gamma band) was closely related to the acquisition of conditioned bradycardia, which indexes the memory of the tone/shock association. Specifically, CS+-induced gamma during the first conditioning session predicted the magnitude of the conditioned response $24 \mathrm{~h}$ later: the greater the gamma during Day 1 of conditioning, the greater the conditioned bradycardia on the following day (Fig. $4 B$, Spearman's rank correlation, $n=21, r=0.67$, $p<0.01)$. However, gamma ceased to predict the next day's performance for subsequent training sessions; that is, gamma on Day 2 did not predict behavior on Day 3, nor did gamma on Day 3 predict behavior on Day 4 (Fig. $4 C, n=20, r=0.18, p>0.1$; Fig. $4 D, n=18, r=0.43, p>0.05)$. Notably, the temporal dynamics of gamma's prediction of behavior paralleled the time course of learning the tone/shock association. Thus, learning oc- 
A

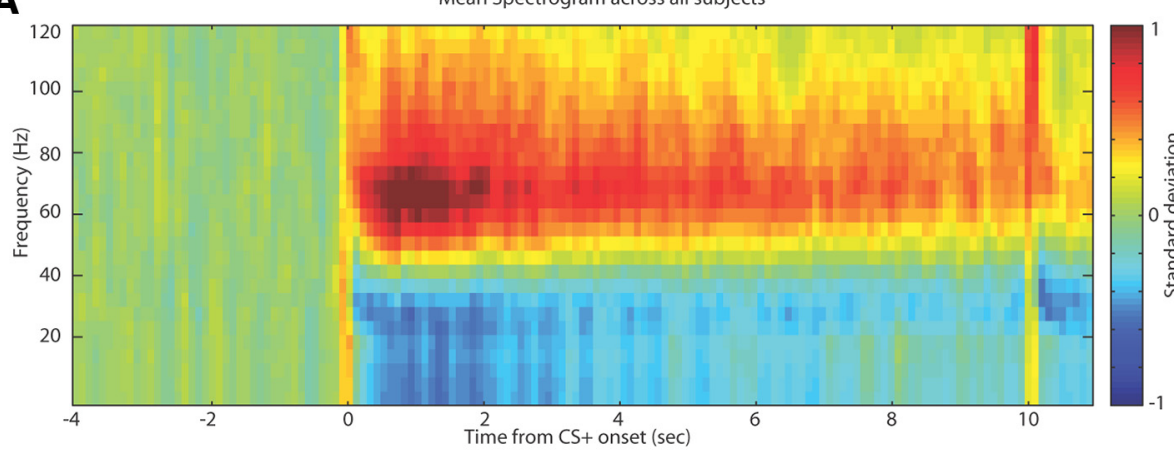

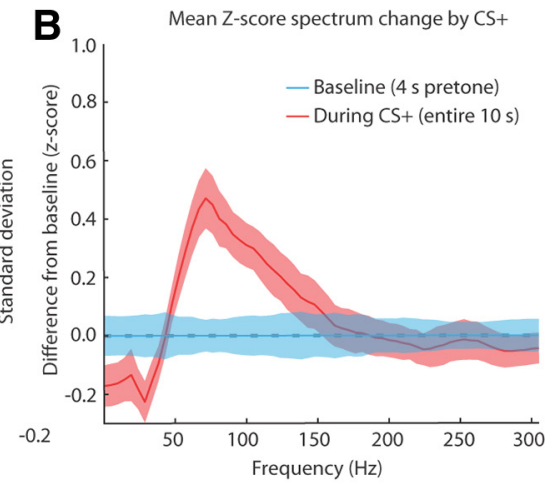

C

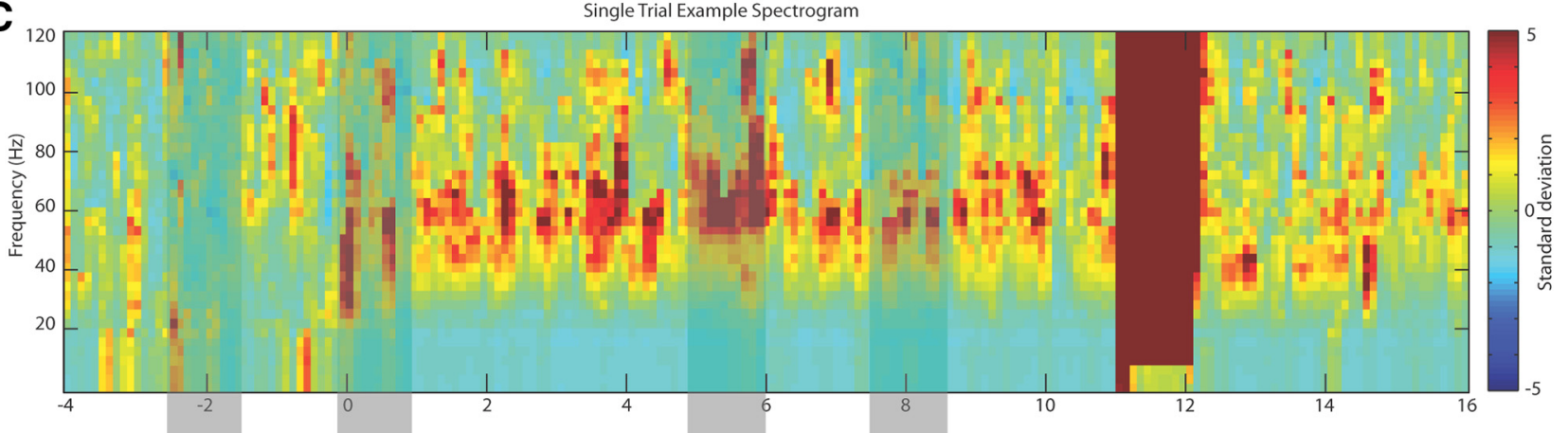

D
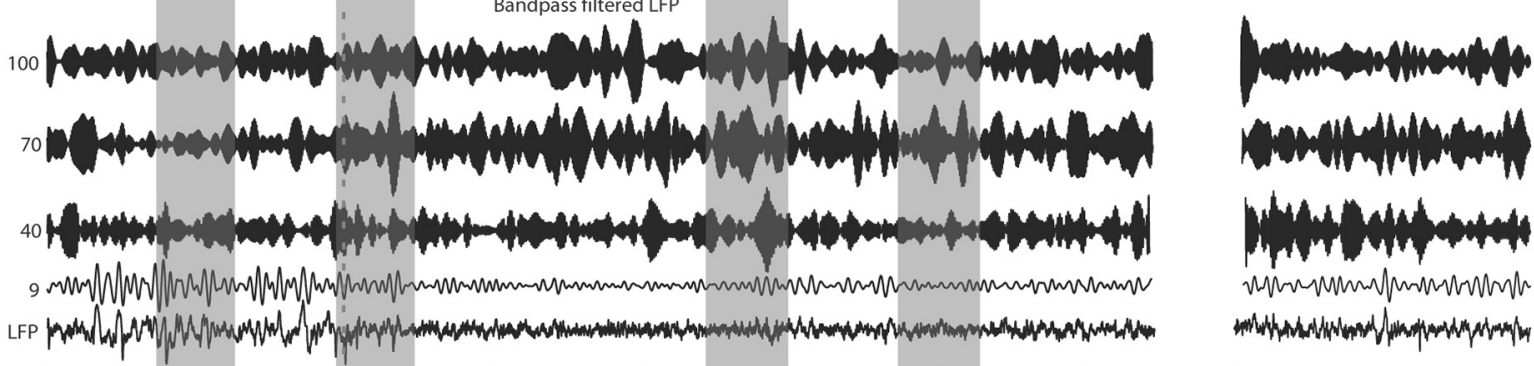

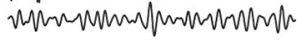

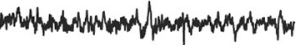

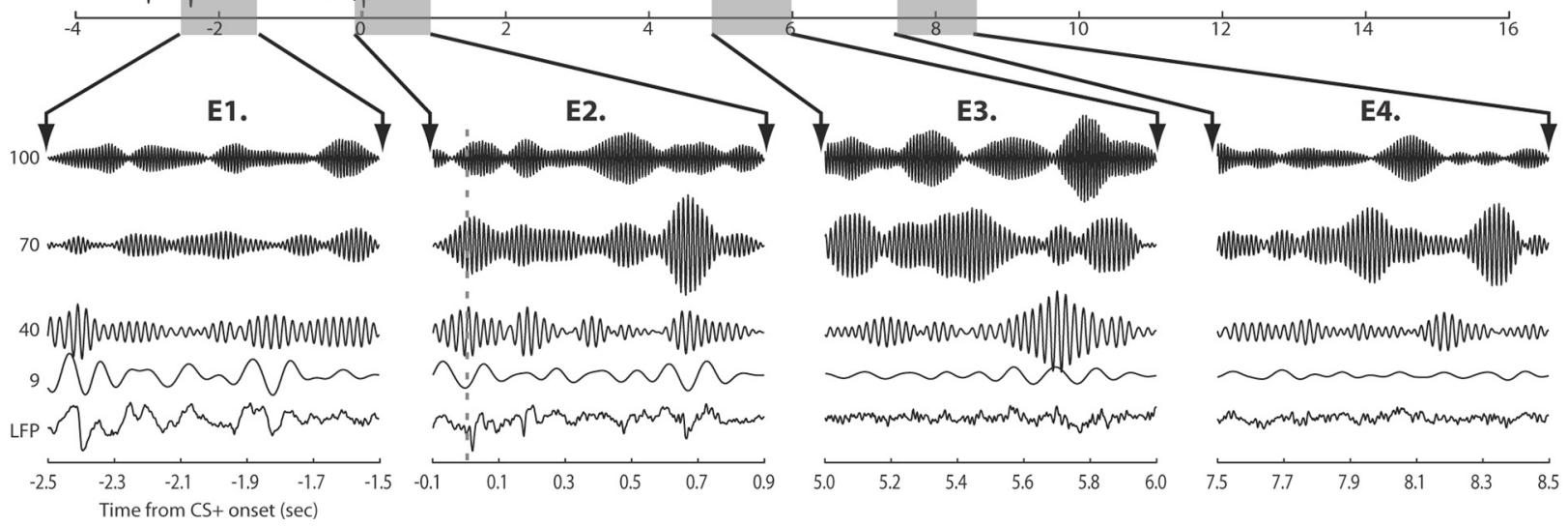

Figure 3. Characterization of the $C S+$-induced gamma activity. $A$, The mean spectrogram from all subjects across all recording sites and conditioning days. A brief broadband increase in spectral power occurs for $200 \mathrm{~ms}$ after the tone, followed by a relatively narrow activation in the gamma band from $40 \mathrm{to} 120 \mathrm{~Hz}$, which develops over several hundred milliseconds and appears to be strongest $0.5-4.0 \mathrm{~s}$ after tone onset. $\boldsymbol{B}$, The mean $z$-score change in spectral power across all recording sites during conditioning. The blue line corresponds to the $4.0 \mathrm{~s}$ pretone baseline period, and the red line is the entire $10 \mathrm{~s}$ tone period. Shaded regions denote $\pm 1 S D$ intervals. $C$, An example spectrogram from a single electrode, on a single trial. Note that gamma activation tends to occur in half-second bursts, which recur throughout the duration of the CS+. The saturated region between 11 and $12 \mathrm{~s}$ was caused by the shock artifact. $D$, Same data as shown in $C$, but instead the LFP waveform and several bandpass ( $10 \mathrm{~Hz}$ bandwidth around each center frequency, Butterworth filter) filtered traces are displayed. The LFP signal displays the hallmarks of low-voltage fast activity after tone onset, and the bandpassed traces show a decrease in power for a low-frequency band $(9 \mathrm{~Hz})$ and an increase in the higher frequency bands $(40,70$, and $100 \mathrm{~Hz})$. Each trace was z-normalized to its pre-CS+ baseline. The gray dotted line indicates the $C S+$ onset. $\boldsymbol{E}$, Four magnified segments of the same data shown in $\boldsymbol{D}$.

curred on Day 1, reached asymptote on Day 2, and did not change thereafter. Similarly, gamma predicted behavior only during this period of learning from Day 1 to Day 2. The concordance of timeframes suggests that gamma's influence on mnemonic processing is restricted to the acquisition of associations.
To further test this hypothesis, we explored an alternative explanation, that subjects with strong induced gamma were predisposed to robust bradycardia, independent of any learning. If so, then subjects with greater induced gamma should display greater bradycardia during the first training session, before the 

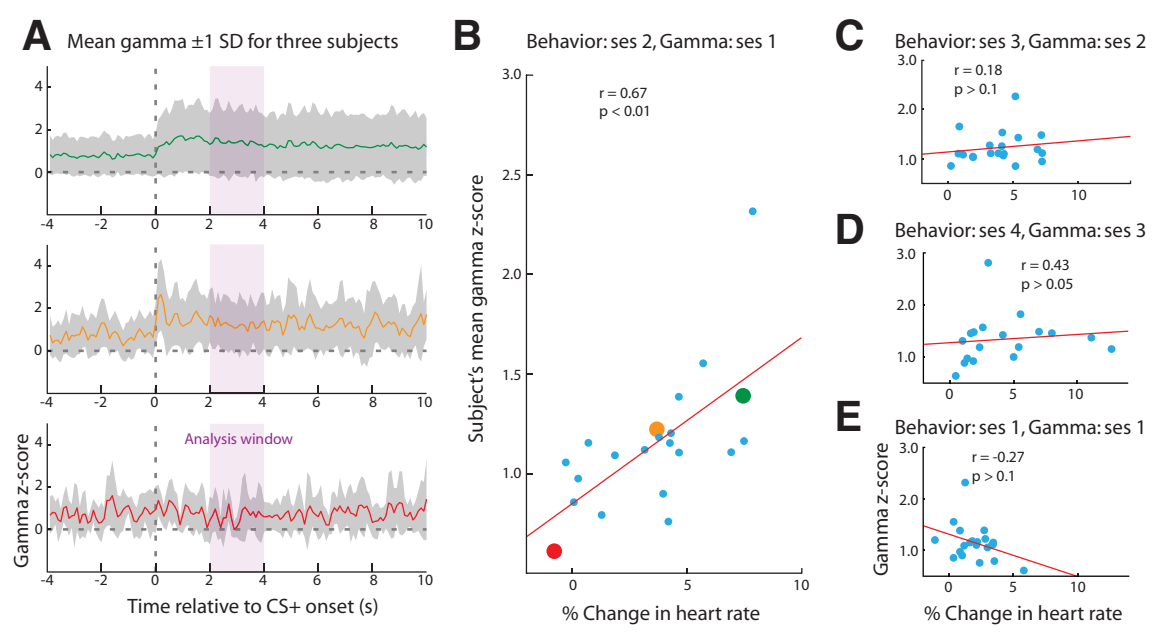

Figure 4. Gamma modulation of memory for the $C S+$ during conditioning. $A$, The $C S+$ on the first day of conditioning elicited gamma-band activation. Several examples of gamma responses from different subjects, in descending order from strongest to weakest. Each colored line is the mean for the first conditioning session, and the shaded gray portion denotes \pm 1 SD across trials. The color of each line indicates the corresponding data point in $\boldsymbol{B}$. The lavender shaded portion of the graph (2-4.0 s from CS+ onset) was the analysis window used to judge induced gamma strength for all analyses. $\boldsymbol{B}, \mathrm{CS}+$-induced gamma during the first conditioning session predicted subsequent behavioral performance on the second session. $\boldsymbol{C}$, The same analysis as in $\boldsymbol{B}$, except that gamma during Session 2 is compared with performance during Session 3. D, Same analysis as in $\boldsymbol{B}$, except that gamma during Session 3 is compared with performance during Session 4 . $\boldsymbol{E}$, The strength of $C S+$-induced gamma during the first conditioning session was compared with CR strength for the same session. For each plot, a straight red line is the best-fit linear regression. All correlations were assessed using Spearman's correlation coefficient. Axes labels in $\boldsymbol{E}$ also apply to $\boldsymbol{C}$ and $\boldsymbol{D}$. second discrimination session and gamma activation to the $\mathrm{CS}-$ on the first discrimination session. No such relationship was found $(n=17, r=$ $-0.38, p>0.1)$. Similar to the CS+ during conditioning, CS--induced gamma also ceased to predict behavioral responding to the $\mathrm{CS}-$ after the first discrimination session (Fig. 5B2, $n=$ $17, r=0.23, p>0.1$; Fig. $5 C 2, n=14$, $r=0.01, p>0.1$ ), nor did gamma predict responding to the CS- within the first training session (Fig. 5D2, $n=19$, $r=-0.04, p>0.1)$.

Thus, gamma also predicts behavior to the CS- only during learning of the new tone contingency (CS--induced gamma on Day 1 of discrimination predicted performance on Day 2). This relationship to behavior mirrors that of gamma and behavior during the initial associative learning on Days 1 and 2 of conditioning. These findings suggest that gamma plays a similar role for the CS + and CS- during initial encoding, specifically, the learning of new information, but not its maintenance. formation of long-term memory. To assess this possibility, we compared the strength of gamma on Day 1 (when it predicted subsequent learning) with performance on that same day. We did not find a significant relationship (Fig. $4 E, n=21, r=$ $-0.27, p>0.1)$.

The cessation of gamma's subsequent memory effect when subjects reached asymptotic performance raises an important issue. Because animals often encounter similar situations, each slightly different from past experience, it should be established whether the mechanisms that facilitate initial learning can return during new learning. We explored this possibility by following the classical conditioning phase with discrimination training. During discrimination training, an unreinforced CS - was introduced, and the CS+ continued to signal shock. Thus, although animals would not learn anything further about the CS + itself, they would learn that a different tone was not followed by shock. Indeed, the onset of discrimination training did not reignite gamma modulation of the next session's behavioral response to the previously learned CS+ (Fig. $5 A 1, n=17, r=0.01, p>0.1$ ). Neither did gamma predict the magnitude of conditioned bradycardia during the subsequent discrimination sessions (Fig. $5 B 1, n=17, r=0.07, p>0.1$; Fig. $5 C 1$, $n=14, r=0.43, p>0.1)$. Thus, the addition of a new training condition, introducing the $\mathrm{CS}-$, did not lead to $\mathrm{CS}+$-induced gamma modulating subsequent performance.

In contrast, $\mathrm{CS}-$-induced gamma on the first discrimination session did predict the strength of bradycardia to the CSduring the second discrimination session. As with its relationship to the CS+ during initial learning in the conditioning phase, increased gamma predicted greater bradycardia to the CS - (Fig. 5A2, $n=17, r=0.51, p<0.05$ ). This correlation may have reflected a relationship between CS--induced gamma and the subsequent discriminability between the CS+ and $\mathrm{CS}-$. Discriminability was measured as the difference in bradycardia between the CS + and $\mathrm{CS}-(\Delta \mathrm{CR}=\mathrm{CS}+\mathrm{CR}-$ CS $-\mathrm{CR})$. We determined the correlation between $\Delta \mathrm{CR}$ on the

\section{Receptive field plasticity}

Given that gamma modulates the initial acquisition of associative memory during conditioning, it may also predict the plasticity that is concomitant with conditioning. Numerous studies have demonstrated CS + frequency-specific receptive field plasticity during conditioning (see Introduction). To characterize longterm $(24 \mathrm{~h})$ plasticity in the primary auditory cortex, we obtained frequency RFs before each training session. The receptive field from the first day of conditioning was treated as the baseline, and those on each subsequent day were each a different posttraining $\mathrm{RF}$. We calculated the percentage change in the RF from each recording site relative to its baseline, yielding "difference RFs" $(\Delta \mathrm{RFs})$.

We found numerous instances of long-term specific receptive field plasticity during classical conditioning (Fig. 6A). Responses to the $\mathrm{CS}+$ frequency were enhanced relative to responses to most other frequencies. As noted (see Introduction), such plasticity may be considered as substrates for mnemonic traces because it has the attributes of associative memory: associativity specificity, rapid acquisition, consolidation, and long-term (months) retention. We also found that responses to the CS were enhanced during classical conditioning, whereas adjacent frequencies did not show any net change.

To determine the specificity of RF plasticity following the first conditioning session, we pooled all the RF changes ( $n=89 \Delta \mathrm{RFs}$, from 35 recording sites within 1.5 octaves of the CS+) across conditioning and tested whether changes at the CS+ or other frequencies within 1.0 octaves were significantly different from zero, indicating a net change (Fig. $6 \mathrm{~B}$, red line). Based on prior studies (Bakin and Weinberger, 1990), we expected to see a significant increase in responding confined to the CS frequency. As found previously, only changes at the CS + frequency were significantly different from zero, with a net increase in responding across the several days of associative training (Fig. $6 \mathrm{~B}$, Wilcoxon signed-rank, $n=89, W=1290, p<0.01)$. None of the changes at 


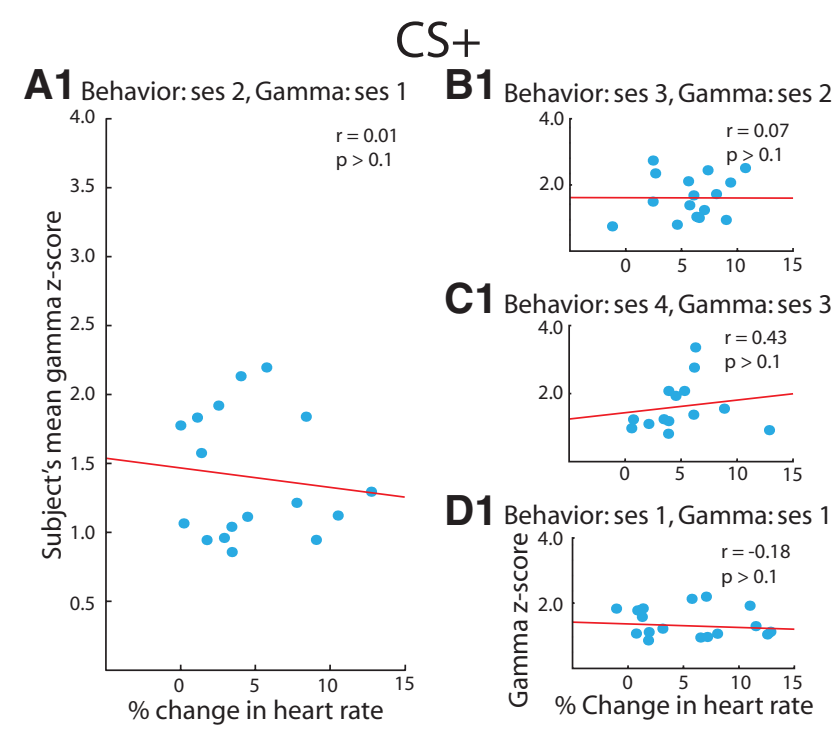

CS-
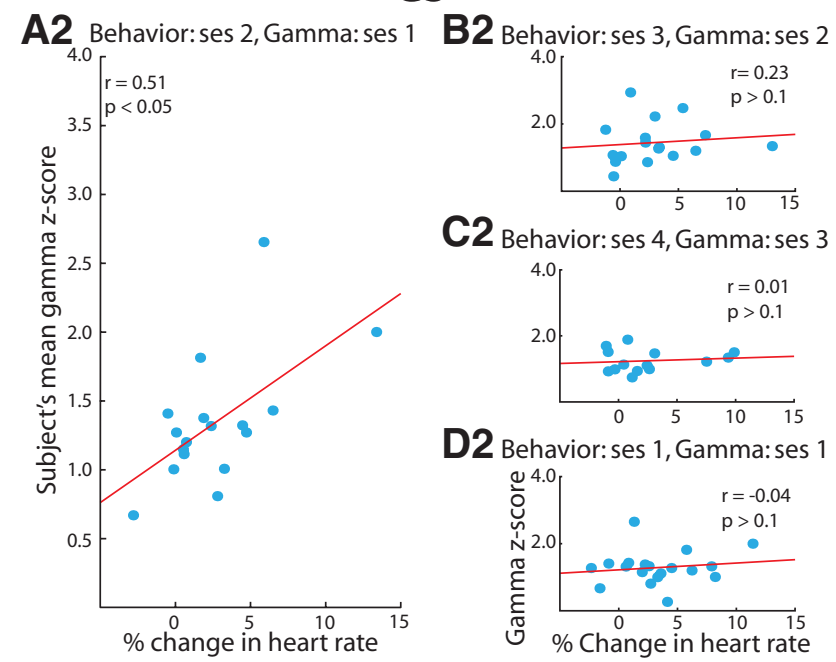

Figure 5. Gamma modulation of memory for the CS + and CS - during discrimination training. Both Sections 1 and 2 of this figure are the same types of comparisons done in Figure 4, but during discrimination training. Section 1 shows the relationship between gamma and performance for the $\mathbf{C S}+$, and Section 2 is the $C S-. A \mathbf{A}, \mathbf{B}$, and $\mathbf{C} \mathbf{1}$ all demonstrate that discrimination training did not revive gamma modulation of responding to the $C S+. D 1$, During the first discrimination session, $\mathrm{CS}+$-induced gamma did not predict performance. This did not mean that gamma had ceased to modulate memory in general, because the gamma induced by the $C S$ - during the first discrimination session correlated with responding to the $C S-$ during the second session, $\boldsymbol{A}$ 2. Similar to the $C S+$ during conditioning, the $C S$--induced gamma correlated with responding to the $C S$ - on the following day, but ceased to correlate with performance for the remaining sessions (B2, $(2)$, and it was not predictive of responding within the first session (D2). All correlations were assessed using Spearman's correlation coefficient. Axes labels in $\boldsymbol{D}$ also apply to $\boldsymbol{B}$ and $\boldsymbol{C}$.

adjacent frequencies were significantly different from zero. Likewise, changes at the CS+ were significantly greater than the pooled changes for frequencies within 1 octave of the CS + (Fig. $6 B$, inset; Wilcoxon rank-sum, $p \approx 0.01$ ).

\section{Gamma modulation of RF plasticity}

Given that gamma predicts behavioral learning, does it also predict the plasticity induced during that learning? To address this issue, we compared the strength of gamma with the magnitude of the RF's percentage change at the CS+ frequency on the following session. We restricted this analysis to recording sites within 1.5 octaves of the CS frequency because of the CS-specific nature of the RF plasticity. By itself, an electrode's gamma strength did not predict the degree of its RF plasticity (Spearman's rank correlation, $n=31, r=0.16, p>0.1)$. However, each subject had a different mean gamma strength, hindering a direct comparison between gamma and plasticity from electrodes pooled across subjects.

To rectify this, gamma strength on each electrode was normalized by dividing the gamma activation on each electrode by the mean gamma for all recording sites with RFs in that subject (henceforth referred to as relative gamma). Thus, electrodes from rats that had very strong gamma could be compared with recording sites from other rats that had weak gamma. This normalization revealed a significant relationship between gamma and plasticity. Specifically, there was an inverted-U relationship between the gamma strength during the first conditioning session and the magnitude of the RF's percentage change at the CS+ frequency $24 \mathrm{~h}$ later, immediately before the second training session. Recording sites with gamma strength near the mean for that subject had the greatest plasticity, whereas those with relatively low or high gamma strength did not display as much plasticity (Fig. $7 A, n=31, r=-0.45, p=0.01$ ).

Similar to gamma modulation of learning during initial training between Conditioning Sessions 1 and 2, gamma predicted plasticity at the CS + frequency. Moreover, gamma on Day 2 of conditioning did not predict plasticity on Day 3 (Fig. $7 B, n=28$, $r=-0.10, p>0.1)$, nor was gamma on Day 3 predictive of plasticity on Day 4 (Fig. 7C, $n=16, r=-0.37, p>0.1$ ).

Potentially, the lack of a relationship between gamma and subsequent plasticity across Sessions 2 and 3 , or 3 and 4 , may arise because $\mathrm{RF}$ changes were assessed relative to the pretraining RF on Day 1. If gamma predicts plasticity from day to day, then a lack of relationship during the later conditioning sessions may be spurious, arising from an improper choice of baseline. To address this issue, we determined the relationship between CS+-induced gamma for a given session and the subsequent between-days variations in RF plasticity. Thus, two new comparisons were performed; gamma on Day 2 was correlated with RF change at the CS + frequency between Days 2 and $3(n=28, r=-0.02, p>0.1)$, and gamma on Day 3 was correlated with RF change between Days 3 and $4(n=16$, $r=0.03, p>0.1)$. The absence of a significant relationship for either test indicates that using the same pretraining baseline for all days does not necessarily limit gamma's modulation of RF plasticity to the initial training session.

In both the current study and others, RF plasticity has been specific to the frequency of the CS+. Consequently, gamma modulation of plasticity also should be specific to the frequency of the CS+. Therefore, we conducted the same analysis for frequencies within \pm 1.0 octaves of the CS + . None of these frequencies displayed plasticity that was modulated by relative gamma on the first training session (Fig. $7 D,-0.25$ octave, $r=-0.07, p>$ $0.1 ;+0.25$ octave, $r=-0.04, p>0.1$; all other frequencies within \pm 1.0 octaves, $p>0.1)$.

To gain a better understanding of the basis for this nonmonotonic relationship between gamma and plasticity, we examined whether three properties of the recording sites covaried with relative gamma strength. The inverted- $U$ relationship between associative plasticity and gamma might have arisen from a relationship between the degree of plasticity and an electrode's octave distance from the CS + frequency: the farther away an electrode's $\mathrm{BF}$ might be from the CS+, the weaker its plasticity. However, if it is too close, then its percentage change could be reduced by already having a strong pretraining response to the CS+. Such dependence could result in a spurious inverted-U relationship, 
A
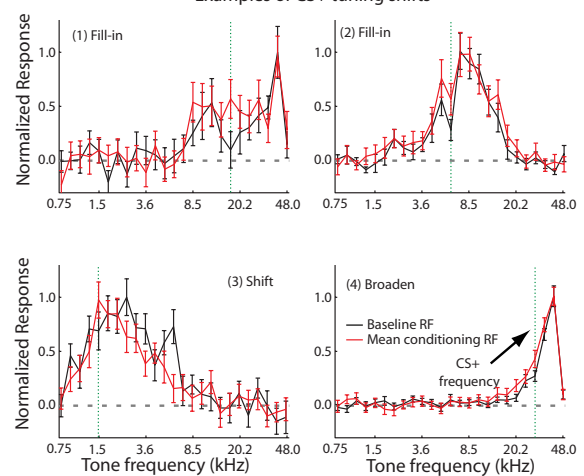

B

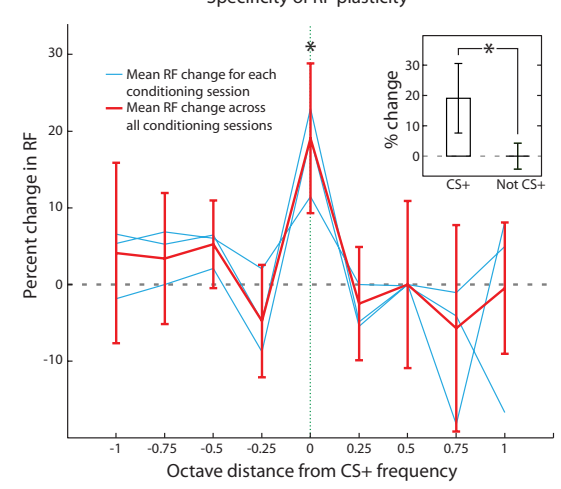

strength and the octave distance of an electrode's BF (peak of tuning) from the CS+. Octave distance of the baseline BF from the $\mathrm{CS}+$ was not related to the normalized gamma strength (Fig. $8 A, n=30, r=0.07$, $p>0.1$.

Another possibility would be that the absolute gamma strength was not monotonically related to relative gamma. In other words, recording sites with stronger relative gamma came from subjects with low overall gamma. Because the mean strength of gamma varied across subjects, and the calculation of relative gamma factored out a subject's mean gamma strength, then the inverted-U function could reflect a difference in gamma strength between subjects and not a relationship common to them all. Thus, we determined whether an electrode's relative gamma strength was related to the magnitude of absolute gamma. Gamma strength before normalization had a significant positive correlation with relative gamma strength (Fig. $8 B, n=30, r=0.48$,

A

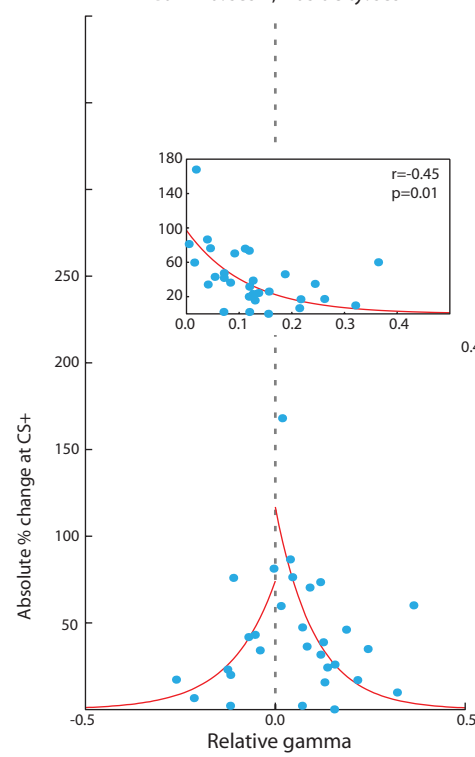

B

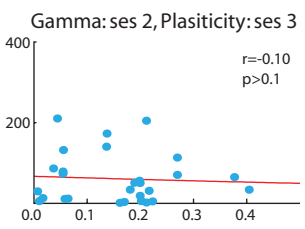

C

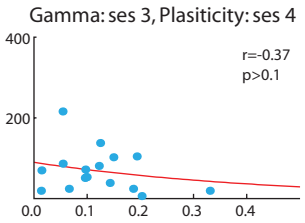

D

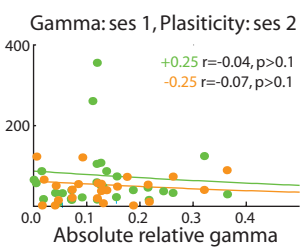

Figure 7. Gamma modulation of plasticity for the $C S+. A, C S+$-induced gamma was normalized relative to the subject's mean gamma. Relative gamma is the percentage difference between gamma on a particular electrode and the mean gamma for that subject. Pooling recording sites across all subjects, relative gamma had an inverted-U relationship with the magnitude of plasticity for the $C S+$. Recording sites with greater absolute relative gamma had weaker plasticity (Spearman's rank correlation, inset graph, $r=-0.45, p \approx 0.01$ ). This relationship between gamma and plasticity at the $C S+$ was only present between relative gamma during the first conditioning session and RF plasticity detected on the subsequent day (B, C). D, To determine whether modulation of RF plasticity was specific to the $C S+$ frequency, we compared relative gamma during the first conditioning session with RF changes \pm 1 octave ( \pm 0.25 octave displayed) away from the $(S+$ on the following day. No relationship was present, indicating a high degree of specificity for gamma modulation of RF plasticity. For all graphs, the regression lines are the best-fit exponential curves. Two data points with outlier values for relative gamma are not shown, but were included for all statistical analyses.

wherein recording sites that are tuned adjacent to the CS+ would have greater relative gamma, but less plasticity. To test whether octave distance caused the apparent prediction of plasticity by gamma, we determined the correlation between relative gamma

$p<0.01$ ), indicating that the nonmonotonic relationship was not an artifact of how relative gamma was calculated.

The third property that we examined was the half-width of mean gamma activation, essentially its bandwidth. Half-width was calculated by finding the peak activation in the gamma-band spectrum, then identifying the bandwidth of the portion of the spectrum that was centered on that peak and had greater than half the peak's strength. Broadband increases in spectral power within the gamma band could reflect nonsynchronous synaptic drive because the LFP reflects the subthreshold membrane potential (Okun et al., 2010), which partly determines action potential generation. If increasing gamma power widens the bandwidth of gamma activation, then any benefit that spike timing-dependent plasticity would derive from an increase in the depth of modulation for subthreshold oscillations would be counteracted by their lack of periodicity (Lee et al., 2009). In our study, gamma bandwidth increased with relative gamma power (Fig. $8 C, n=30, r=$ $0.37, p<0.05)$, indicating that stronger relative gamma may lack the synchrony necessary to effectively induce plasticity. Broadband gamma oscillations would reflect asynchronous activity, which would be less likely to promote the well timed presynaptic and postsynaptic activity necessary for synaptic change (Migliore and Lansky, 1999). Thus, whereas weaker relative gamma lacks sufficient depth of modulation, stronger relative gamma, which is correlated with increased spectral bandwidth, lacks coherence.

Therefore, the predictive relationship between gamma and specific plasticity in A1 was not a byproduct of a recording site's octave distance from the CS frequency or the calculation of relative gamma. Instead, it may reflect the relationship between the bandwidth and strength of gamma activation, with stronger gamma having a broader bandwidth and consequently less synchronous activation.

\section{Discussion}

An overriding question in behavioral neuroscience is how experiences modify future performance and concordantly impact and shape neural circuitry. Presumably, both of these modifications are mediated by the same mechanisms because neural plasticity is the basis for lasting changes in behavior. Gamma-band oscillations are one such mechanism because they are predictive of 
subsequent memory for stimuli and have been implicated in the modulation of synaptic plasticity in vitro. To experimentally unify these two research programs, we determined in the same subjects, under the same training paradigm, whether gamma oscillations induced by training stimuli are correlated with both long-term memory and experience-dependent specific plasticity. Our results indicate that gamma significantly predicts both during initial learning.

\section{Gamma and memory strength}

Gamma oscillation strength predicted the acquisition of the CS+/shock association.

The strength of CS+-induced gamma-band activation during the first conditioning session correlated with the degree of responding to the CS+ on the second session, at which behavioral asymptote was attained. After the first training session, gamma ceased to predict subsequent memory. Even the onset of discrimination training did not revive the relationship between gamma and responding to the CS+. This time-limited role of gamma oscillations suggests that their function is not the maintenance of memories. However, other investigators have provided experimental and theoretical support for oscillations in the beta band $(15-30 \mathrm{~Hz})$ serving that function (Engel and Fries, 2010; Kopell et al., 2011).

In contrast to the $\mathrm{CS}+, \mathrm{CS}-$-induced gamma on the first day of discrimination did predict responding to the CS- on the second discrimination session. CS--induced gamma was related to future CS- responding in the same manner as the CS+. Interestingly, the CS- was never paired with a shock, suggesting that induced gamma does not necessarily promote veridical memories. Neural phenomena that exhibit subsequent memory effects should display the same phenomenology during encoding for both true and false memories (Gonsalves and Paller, 2000). Gamma induced by the CS - showed this relationship; subjects that had stronger gamma activity induced by the CS - during the first discrimination session responded with greater bradycardia to it during the second session (Fig. 5A2). Also, like the CS+ during conditioning, only gamma induced by the CS- during the first session of discrimination predicted performance on the subsequent session.

This finding has several possible explanations. Gamma may have enhanced CS - encoding, facilitating CS - recall on the second discrimination session independent of any particular association. Enhanced recall could have driven an inference that the CS - had been paired with a shock (Foote and Crystal, 2007). Another possibility is that gamma induced by the CS - reflected arousal during training, and this heightened emotional state was associated with the CS- (Dickinson and Balleine, 1994). Similarly, stronger CS - gamma might have derived from an expectancy of shock, generating a conjunction, which was later retrieved as an association between the tone and shock. Although further work is necessary to distinguish between these hypotheses, one can conclude that increased gamma does not necessarily promote accurate memory.

The examined duration and latency of this gamma modulation, 2-4 s after CS onset, may reflect the action of neuromodulatory systems on auditory cortex. One such neuromodulator, $\mathrm{ACh}$, affects both gamma synchrony and memory. The time course of ACh release is similar to that for induced gamma in this
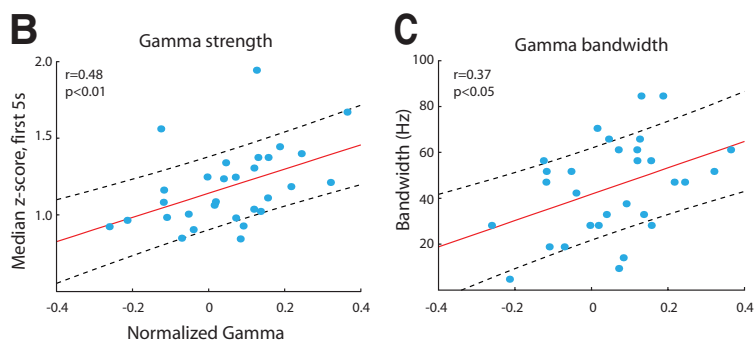
affect gamma (Buhl et al., 1998). Cholinergic agonists can enhance stimulus-induced gamma oscillations in visual cortex, whereas the muscarinic antagonist scopolamine reduces those oscillations (Rodriguez et al., 2004). With regard to memory, $\mathrm{ACh}$ is released in A1 during auditory classical conditioning (Butt et al., 2009), lesioning structures containing Ach-releasing neurons prevent learning, and greater $\mathrm{ACh}$ release facilitates learning (for review, see Pepeu and Giovannini, 2004).

\section{Gamma prediction of CS+ frequency-specific plasticity}

As gamma-band activity induced by a CS predicts the subsequent memory for that same stimulus, gamma might also modulate attendant plasticity. To investigate this relationship, we tracked changes in A1 receptive fields across several days of associative training. RF plasticity was specific to the CS+; only at the CS+ frequency were changes in the RF significantly different from zero, unlike all other frequencies within 1.0 octave of the CS+.

Specific plasticity was, indeed, related to gamma activity. Thus, gamma predicted both memory strength and the specific plasticity that formed during the task. However, these findings do not imply a simple relationship between associative memory and plasticity. Associative learning in the form of classical (Pavlovian) conditioning may appear to be simple because it involves one stimulus preceding another, "reflex-like." However, conditioning is highly cognitive, involving continual assessments about the probabilistic fabric of the environment (Rescorla, 1988).

The magnitude of plasticity had an inverted-U relationship with gamma strength. Specifically, recordings with relatively weak or strong gamma showed less plasticity than those recording sites that were near the mean gamma strength for that subject. Like gamma modulation of memory, CS+-induced gammaband activation only modulated plasticity produced after the first training session. Gamma on each subsequent conditioning session did not predict plasticity detected on the following day. This modulation of plasticity was also specific to the CS+ frequency: none of the frequencies within 1.0 octave of the CS+ showed a modulation of their percentage change by gamma.

The inverted-U relationship between gamma and plasticity was unexpected. Prior theorizing on gamma oscillations and plasticity have not discussed the possibility that enhanced gamma power could depress plasticity (Traub et al., 1998; Axmacher et al., 2006; Wang, 2010). But our results are explicable if gamma reflects the action of ACh. RF plasticity is preferentially induced during cortical desynchronization, a brain state that has been linked to ACh release (Metherate et al., 1992; Cruikshank and Weinberger, 1996). With regard to the inverted-U relationship, 
carbachol, a muscarinic receptor agonist, enhances hippocampal and cortical LTP at moderate doses, but reduces LTP at higher doses (Shimoshige et al., 1997; Dringenberg et al., 2007). A similar trend occurs for cholinergic modulation of attentional plasticity. Iontophoretically applied ACh enhances attentional modulation of RFs in visual cortex, but only for low dosages (Herrero et al., 2008). Increasing ACh application either had no modulatory effect or slightly reduced RF modulation. Given the coupling between gamma oscillations and the cholinergic system, an inverted-U relationship between gamma and plasticity is comprehensible.

The role of ACh in learning-induced specific auditory RF plasticity and associative memory has already been established. Cholinergic blockade impairs conditioning-induced specific plasticity in the human auditory cortex (Thiel et al., 2002). Cholinergic innervation of the cortex is supplied by the nucleus basalis (NB) (Mesulam et al., 1983), which is activated during pairing of a cue with reinforcement (Wilson and Rolls, 1990). ACh is released in the primary auditory cortex during classical conditioning (Butt et al., 2009). Pairing a tone with stimulation of the NB induces CS-specific RF plasticity (Bakin et al., 1996; Kilgard et al., 2001). Furthermore, this plasticity has the same attributes as the RF plasticity that develops during normal associative learning, that is, associativity, specificity, rapid development, consolidation, and long-term retention (Weinberger, 1998; Galván and Weinberger, 2002). Finally, a tone paired with NB stimulation also induces actual CS-specific associative behavioral memory (McLin et al., 2002), and this "implanted memory" also has the cardinal attributes of natural memory (e.g., Miasnikov et al., 2011) and is dependent on muscarinic receptors (Miasnikov et al., 2008).

A model of CS-specific RF plasticity posits that ascending auditory and shock information from the ventral and magnocellular medial geniculate (MGm) nuclei, respectively, coincides with the release of $\mathrm{ACh}$ in $\mathrm{A} 1$, from $\mathrm{NB}$ that is activated from the $\mathrm{MGm}$ via the amygdala (Weinberger et al., 1990). Subsequent studies have provided strong support for this model in humans (Morris et al., 1998) and animals (Weinberger, 1998), but a link between the release of ACh and cortical plasticity had been missing. The current findings appear to provide this link because the release of ACh enhances gamma (Rodriguez et al., 2004), and gamma predicts CS-specific RF plasticity in A1.

\section{Conclusion}

Gamma oscillations are thought to play a role in memory formation and experience-dependent plasticity (Jutras and Buffalo, 2010; Fell and Axmacher, 2011). This study demonstrates that gamma-band oscillations, or at least the array of neural phenomena they reflect, predict both long-term memory and plasticity under the same circumstances. The current findings implicate gamma in memory formation because it is predictive of initial associative learning and modulates cortical receptive field plasticity with a high degree of specificity.

The specificity of gamma activation for modulating memory and plasticity stands in contrast to the ubiquity of gamma oscillations across training sessions and cortical sites. Why does gamma only modulate subsequent performance during acquisition of the association, and why does its modulatory reach not extend to plasticity at non-CS frequencies? One answer is that gamma reflects a variety of neural phenomena, each with a unique role and functional consequence (Wang, 2010). Gamma on the first day of training, or for those populations representing the CS + frequency, could be fundamentally different from gamma produced later in training or at non-CS + brain sites. Understanding this shifting mechanistic basis behind the generation and impact of gamma rhythms will greatly promote our understanding of the operation of the brain in general.

\section{References}

Axmacher N, Mormann F, Fernández G, Elger CE, Fell J (2006) Memory formation by neuronal synchronization. Brain Res Rev 52:170-182.

Bakin JS, Weinberger NM (1990) Classical conditioning induces CSspecific receptive field plasticity in the auditory cortex of the guinea pig. Brain Res 536:271-286.

Bakin JS, South DA, Weinberger NM (1996) Induction of receptive field plasticity in the auditory cortex of the guinea pig during instrumental avoidance conditioning. Behav Neurosci 110:905-913.

Bi GQ, Poo MM (1998) Synaptic modifications in cultured hippocampal neurons: dependence on spike timing, synaptic strength, and postsynaptic cell type. J Neurosci 18:10464-10472.

Bieszczad KM, Weinberger NM (2010) Representational gain in cortical area underlies increase of memory strength. Proc Natl Acad Sci U S A 107:3793-3798.

Bokil H, Andrews P, Kulkarni JE, Mehta S, Mitra PP (2010) Chronux: a platform for analyzing neural signals. J Neurosci Methods 192:146-151.

Bouton ME (2006) Learning and behavior: a contemporary synthesis, Ed 1. Sunderland, MA: Sinauer.

Buhl EH, Tamas G, Fisahn A (1998) Cholinergic activation and tonic excitation induce persistent gamma oscillations in mouse somatosensory cortex in vitro. J Physiol 513(Pt 1):117-126.

Butt AE, Chavez CM, Flesher MM, Kinney-Hurd BL, Araujo GC, Miasnikov AA, Weinberger NM (2009) Association learning-dependent increases in acetylcholine release in the rat auditory cortex during auditory classical conditioning. Neurobiol Learn Mem 92:400-409.

Buzsáki G (2006) Rhythms of the brain. New York: Oxford UP.

Chrobak JJ, Buzsáki G (1998) Gamma oscillations in the entorhinal cortex of the freely behaving rat. J Neurosci 18:388-398.

Cruikshank SJ, Weinberger NM (1996) Receptive-field plasticity in the adult auditory cortex induced by Hebbian covariance. J Neurosci 16:861-875.

Diamond DM, Weinberger NM (1989) Role of context in the expression of learning-induced plasticity of single neurons in auditory cortex. Behav Neurosci 103:471-494.

Dickinson A, Balleine B (1994) Motivational control of goal-directed action. Learn Behav 22:1-18.

Dringenberg HC, Hamze B, Wilson A, Speechley W, Kuo MC (2007) Heterosynaptic facilitation of in vivo thalamocortical long-term potentiation in the adult rat visual cortex by acetylcholine. Cereb Cortex 17:839-848.

Engel AK, Fries P (2010) Beta-band oscillations-signalling the status quo? Curr Opin Neurobiol 20:156-165.

Fell J, Axmacher N (2011) The role of phase synchronization in memory processes. Nat Rev Neurosci 12:105-118.

Fell J, Klaver P, Lehnertz K, Grunwald T, Schaller C, Elger CE, Fernández G (2001) Human memory formation is accompanied by rhinal-hippocampal coupling and decoupling. Nat Neurosci 4:1259-1264.

Fendt M, Fanselow MS (1999) The neuroanatomical and neurochemical basis of conditioned fear. Neurosci Biobehav Rev 23:743-760.

Foote AL, Crystal JD (2007) Metacognition in the rat. Curr Biol 17:551-555.

Fritz J, Shamma S, Elhilali M, Klein D (2003) Rapid task-related plasticity of spectrotemporal receptive fields in primary auditory cortex. Nat Neurosci 6:1216-1223

Froemke RC, Merzenich MM, Schreiner CE (2007) A synaptic memory trace for cortical receptive field plasticity. Nature 450:425-429.

Galván VV, Weinberger NM (2002) Long-term consolidation and retention of learning-induced tuning plasticity in the auditory cortex of the guinea pig. Neurobiol Learn Mem 77:78-108.

Goldstein MH Jr, Abeles M, Daly RL, McIntosh J (1970) Functional architecture in cat primary auditory cortex-tonotopic organization. J Neurophysiol 33:188-197.

Gonsalves B, Paller KA (2000) Neural events that underlie remembering something that never happened. Nat Neurosci 3:1316-1321.

Herrero JL, Roberts MJ, Delicato LS, Gieselmann MA, Dayan P, Thiele A (2008) Acetylcholine contributes through muscarinic receptors to attentional modulation in V1. Nature 454:1110-1114.

Isaac JT, Buchanan KA, Muller RU, Mellor JR (2009) Hippocampal place 
cell firing patterns can induce long-term synaptic plasticity in vitro. J Neurosci 29:6840-6850.

Jutras MJ, Buffalo EA (2010) Synchronous neural activity and memory formation. Curr Opin Neurobiol 20:150-155.

Jutras MJ, Fries P, Buffalo EA (2009) Gamma-band synchronization in the macaque hippocampus and memory formation. J Neurosci 29:1252112531.

Kilgard MP, Pandya PK, Vazquez J, Gehi A, Schreiner CE, Merzenich MM (2001) Sensory input directs spatial and temporal plasticity in primary auditory cortex. J Neurophysiol 86:326-338.

Kopell N, Whittington MA, Kramer MA (2011) Neuronal assembly dynamics in the betal frequency range permits short-term memory. Proc Natl Acad Sci U S A 108:3779-3784.

Lee S, Sen K, Kopell N (2009) Cortical gamma rhythms modulate NMDARmediated spike timing dependent plasticity in a biophysical model. PLoS Comput Biol 5:e1000602.

Lutzenberger W, Ripper B, Busse L, Birbaumer N, Kaiser J (2002) Dynamics of gamma-band activity during an audiospatial working memory task in humans. J Neurosci 22:5630-5638.

McLin DE 3rd, Miasnikov AA, Weinberger NM (2002) Induction of behavioral associative memory by stimulation of the nucleus basalis. Proc Natl Acad Sci U S A 99:4002-4007.

Mesulam MM, Mufson EJ, Levey AI, Wainer BH (1983) Cholinergic innervation of cortex by the basal forebrain: cytochemistry and cortical connections of the septal area, diagonal band nuclei, nucleus basalis (substantia innominata), and hypothalamus in the rhesus monkey. J Comp Neurol 214:170-197.

Metherate R, Cox CL, Ashe JH (1992) Cellular bases of neocortical activation: modulation of neural oscillations by the nucleus basalis and endogenous acetylcholine. J Neurosci 12:4701-4711.

Miasnikov AA, Chen JC, Weinberger NM (2008) Specific auditory memory induced by nucleus basalis stimulation depends on intrinsic acetylcholine. Neurobiol Learn Mem 90:443-454.

Miasnikov AA, Chen JC, Weinberger NM (2011) Consolidation and longterm retention of an implanted behavioral memory. Neurobiol Learn Mem 95:286-295.

Migliore M, Lansky P (1999) Long-term potentiation and depression induced by a stochastic conditioning of a model synapse. Biophys J 77:1234-1243.

Morris JS, Friston KJ, Dolan RJ (1998) Experience-dependent modulation of tonotopic neural responses in human auditory cortex. Proc Biol Sci 265:649-657.

Okun M, Naim A, Lampl I (2010) The subthreshold relation between cortical local field potential and neuronal firing unveiled by intracellular recordings in awake rats. J Neurosci 30:4440-4448.

Osipova D, Takashima A, Oostenveld R, Fernández G, Maris E, Jensen O (2006) Theta and gamma oscillations predict encoding and retrieval of declarative memory. J Neurosci 26:7523-7531.

Paller KA, Wagner AD (2002) Observing the transformation of experience into memory. Trends Cogn Sci 6:93-102.

Parikh V, Kozak R, Martinez V, Sarter M (2007) Prefrontal acetylcholine release controls cue detection on multiple timescales. Neuron 56:141154.

Pepeu G, Giovannini MG (2004) Changes in acetylcholine extracellular levels during cognitive processes. Learn Mem 11:21-27.

Pesaran B, Pezaris JS, Sahani M, Mitra PP, Andersen RA (2002) Temporal structure in neuronal activity during working memory in macaque parietal cortex. Nat Neurosci 5:805-811.
Reale RA, Imig TJ (1980) Tonotopic organization in auditory cortex of the cat. J Comp Neurol 192:265-291.

Rescorla RA (1988) Pavlovian conditioning. It's not what you think it is. Am Psychol 43:151-160.

Rodriguez R, Kallenbach U, Singer W, Munk MH (2004) Short- and longterm effects of cholinergic modulation on gamma oscillations and response synchronization in the visual cortex. J Neurosci 24:10369-10378.

Rutkowski RG, Weinberger NM (2005) Encoding of learned importance of sound by magnitude of representational area in primary auditory cortex. Proc Natl Acad Sci U S A 102:13664-13669.

Sally SL, Kelly JB (1988) Organization of auditory cortex in the albino rat: sound frequency. J Neurophysiol 59:1627-1638.

Sederberg PB, Gauthier LV, Terushkin V, Miller JF, Barnathan JA, Kahana M] (2006) Oscillatory correlates of the primacy effect in episodic memory. Neuroimage 32:1422-1431.

Sederberg PB, Schulze-Bonhage A, Madsen JR, Bromfield EB, McCarthy DC, Brandt A, Tully MS, Kahana MJ (2007) Hippocampal and neocortical gamma oscillations predict memory formation in humans. Cereb Cortex 17:1190-1196.

Shimoshige Y, Maeda T, Kaneko S, Akaike A, Satoh M (1997) Involvement of M2 receptor in an enhancement of long-term potentiation by carbachol in Schaffer collateral-CA1 synapses of hippocampal slices. Neurosci Res 27:175-180.

Siegel M, König P (2003) A functional gamma-band defined by stimulusdependent synchronization in area 18 of awake behaving cats. J Neurosci 23:4251-4260.

Siegel S, Castellan NJ (1988) Nonparametric statistics for the behavioral sciences, Ed 2. New York: McGraw-Hill.

Teyler TJ (1971) Effects of restraint on heart-rate conditioning in rats as a function of US location. J Comp Physiol Psychol 77:31-37.

Thiel CM, Friston KJ, Dolan RJ (2002) Cholinergic modulation of experience-dependent plasticity in human auditory cortex. Neuron 35:567-574.

Thomson DJ (1982) Spectrum estimation and harmonic analysis. Proc IEEE 70:1055-1096.

Traub RD, Spruston N, Soltesz I, Konnerth A, Whittington MA, Jefferys GR (1998) Gamma-frequency oscillations: a neuronal population phenomenon, regulated by synaptic and intrinsic cellular processes, and inducing synaptic plasticity. Prog Neurobiol 55:563-575.

Wang XJ (2010) Neurophysiological and computational principles of cortical rhythms in cognition. Physiol Rev 90:1195-1268.

Weinberger NM (1998) Physiological memory in primary auditory cortex: characteristics and mechanisms. Neurobiol Learn Mem 70:226-251.

Weinberger NM (2004) Specific long-term memory traces in primary auditory cortex. Nat Rev Neurosci 5:279-290.

Weinberger NM, Ashe JH, Metherate R, McKenna TM, Diamond DM, Bakin J (1990) Retuning auditory cortex by learning: a preliminary model of receptive field plasticity. Concept Neurosci 1:91-132.

Wespatat V, Tennigkeit F, Singer W (2004) Phase sensitivity of synaptic modifications in oscillating cells of rat visual cortex. J Neurosci 24:90679075.

Wilson FA, Rolls ET (1990) Learning and memory is reflected in the responses of reinforcement-related neurons in the primate basal forebrain. J Neurosci 10:1254-1267.

Winters RW, McCabe PM, Schneiderman N (2002) Functional utility and neurobiology of conditioned autonomic responses. In: A neuroscientist's guide to classical conditioning (Moore JW, ed), pp 46-85. New York: Springer. 